\title{
¿ Existe el cáncer localizado de próstata?
}

\author{
¿Does cancer localized to the prostate gland really exist? \\ LOBEL MANDEL Bernard ${ }^{1}$, GUILLE SENDRALES Francois ${ }^{2}$, PATARD ROULIER Jean-Jacques ${ }^{3}$, ZEGARRA \\ MONTES Luis ${ }^{4}$.
}

\begin{abstract}
SUMMARY
The natural history of prostate cancer is not well defined and this further complicates the therapeutic options for its treatment. Since 1980, radical prostatectomy was considered the first option in attempting to cure this type of cancer which represents a $25 \%$ of the cases of cancer in men, affecting $1 / 8$ of the male population before age 75 and that reveals itself as the more frequent male cancer after age 50. Due to its effectiveness, radical prostatectomy should be performed when cancer is limited to the gland, not having surpassed the prostatic gland. The acquired experience shows that 20$40 \%$ of anatomical pieces extracted presented capsular invasion or positive margins, and that approximately $30-60 \%$ of the operated patients witness the progress of their disease within 5 years, despite surgery. This thought is possible due to the acquired experience with numerous patients that have been treated with radical prostatectomy. All the recent work done confirms that only cancer localized to the prostatic gland is susceptible of being cured by surgery and that only low risk cancers have a real possibility of being limited to the gland. In this group of patients with localized cancer, many are not of clinical importance. An important number of patients that have neoplasic cells in their prostate will die from other causes, without having necessarily suffered from the prostate. This paper highlights the limits of surgery and the actual lack of criteria to define a localized lesion within the gland and its aggressiveness, as well as the lack of alternative effective treatments in the case of an extra prostatic lesion. Finally, it is very important to try to make an early diagnosis of prostatic cancer, nevertheless, from the moment in which the diagnosis of cancer is given to the patient, the single word "cancer" sounds devastating and its announcement transforms the patient's life, with an upcoming death sensation that could pose a psychological harm to the patient. Just to mention, for the urologist not to over estimate cancer and not to over treat it with interventions that may definitely alter the quality of life of the patient: radical prostatectomy causes urinary incontinence in 5-20\% of patients and erectile disfunction in $40-70 \%$ of them. $\&$ Does cancer localized to the prostate gland really exist? The question arises now between the cases of cancer that are diagnosed while they are not significative and the ones that have already surpassed the capsule. (Rev Med Hered 2005; 16: 202-207)
\end{abstract}

KEY WORDS: Prostate, cancer localized.

\section{INTRODUCCIÓN}

La historia natural del cáncer de próstata no se conoce bien, lo cual hace difícil las elecciones terapéuticas para combatirlo. Desde 1980, la prostatectomía radical se impuso para intentar curar este cáncer que representa un $25 \%$ de casos de cáncer en el hombre, afectando a $1 / 8$ antes de los 75 años y que se revela como el cáncer masculino más frecuente después de los 50 años. Por ser eficaz, la prostatectomía radical debe de realizarse

Jefe Servicio Urología-Hospital Universitario Pontchaillou, Rennes-Francia.

Profesor Honorario Universidad Peruana Cayetano Heredia.

2 Urólogo Asistente-Hospital Universitario Pontchaillou, Rennes-Francia. Decano Facultad Medicina- Universidad Rennes-Francia

Urólogo Asistente - Hospital Universitario Pontchaillou, Rennes-Francia

Jefe Servicio Urología-Hospital Nacional Cayetano Heredia. Profesor Principal del Departamento de CirugíaUniversidad Peruana Cayetano Heredia. 
cuando el cáncer se limita a la glándula, no habiendo sobrepasado la cápsula prostática. La experiencia adquirida demostró que 20 a $40 \%$ de piezas anatómicas extraídas presentan una invasión capsular o márgenes positivos y que de 30 a $60 \%$ de los pacientes operados ven que su enfermedad progresa en 5 años a pesar de la cirugía.

Hoy en día surgen dos preguntas: ¿ Existe el cáncer de próstata localizado? ¿ La prostatectomía radical, cuyas consecuencias sobre la continencia urinaria y la sexualidad conocemos, merece el lugar que le damos actualmente?

Esta reflexión es posible gracias a la experiencia adquirida con numerosos enfermos que han sido tratados a través de prostatectomía radical.

\section{Definir el cáncer localizado de próstata}

En la actualidad para hablar de cáncer de próstata localizado se requieren 2 criterios: uno anatómico, dado por el examen anatomopatológico y el otro aportado por las informaciones en relación a la noción de un cáncer de bajo riesgo.

\section{Criterio anatomopatológico}

El cáncer de próstata localizado debe limitarse a la glándula y no invadir ni la pseudocápsula que la separa del tejido céluloadiposo periprostático, ni del ápex de la glándula que está desprovisto de cápsula, ni del cuello de la vejiga o las vesículas seminales. Este cáncer localizado a la próstata, es en principio tratado a través de prostatectomía radical que lo extrae en su totalidad. En otros casos, el cáncer se considera evolutivo por lo tanto ya no se justifica una intervención quirúrgica que pretende ser curativa $\left(\right.$ Tabla $\left.\mathrm{N}^{\circ} 1\right)$.

¿Cómo asegurarse que el cáncer tiene carácter limitado a la glándula antes de la prostatectomía radical? El tacto rectal no es suficiente para afirmarlo ya que utilizado solo, es impreciso en un $60 \%$ de los casos, ya sea por razones de sobrediagnóstico o subdiagnóstico. La ecografía endorectal ya no es efectiva $(50 \%$ de sensibilidad y $40 \%$ a $70 \%$ de especificidad). Las biopsias de próstata son más pertinentes pero subestiman la presencia de cáncer o su grado en $30 \%$ de los casos y lo sobreestiman de 4 a 30\% de los casos. La resonancia magnética nuclear está en estudio y podría adaptarse.

Cualquiera que sea el método de diagnóstico, debemos de reconocer el carácter imprevisible de los resultados del examen anatomopatológico después de una prostatectomía radical. De esta manera Polascik (7) constata en una cohorte de 4000 pacientes enviados por cáncer de próstata próximos a someterse a una prostatectomía radical, que el $50 \%$ son rechazados por una lesión localmente avanzada o metastásica y que del $50 \%$ de operados, solamente la mitad $(25 \%$ de la cohorte) presenta lesión intraglandular (o localizada) en el examen anatomopatológico, mientras que 15\% presenta una infiltración capsular y 10\% presenta márgenes operatorios positivos.

\section{La noción del riesgo}

Partin (3) desde 1997, estudia una cohorte de más de 4000 pacientes operados de prostatectomía radical y describe los criterios de estadío patológico final frente a un tumor aparentemente localizado. Epstein (1) ya había precisado en 1994 los criterios clínicos y patológicos que ante un cáncer impalpable descubierto en la biopsia de próstata practicada en una evaluación Antígeno Prostático Específico (APE) (estadío T1c), permitían prever la extensión tumoral fuera de la glándula. Dichos criterios se establecen sobre el grado de Gleason (nivel de diferenciación del tumor), la densidad del APE (tasa de APE relacionada al volumen de la glándula) y los caracteres de infiltración tumoral sobre las biopsias. De este modo, los tumores de bajo riesgo o limitados a la glándula están definidos por una densidad de APE inferior a $0,1 \mathrm{ng} / \mathrm{ml} / \mathrm{g}$ y la ausencia de un factor de gravedad sobre la biopsia (score de Gleason

Tabla N¹. Definición del cancer avanzado de prostata.

\section{Cáncer avanzado}

Localmente avanzado

Avanzado

\section{Definición}

No confinado al órgano

No metastásico

Recurrencia local después de Rt.

Todo tipo de extensión $(\mathrm{N}+, \mathrm{M}+)$

Respuesta (-) a terapia hormonal

Rt : Radioterapia 
Figura $N^{\circ} 1$. Correlación del score de Gleason y resultado anatomopatológico post-prostatectomía radical.

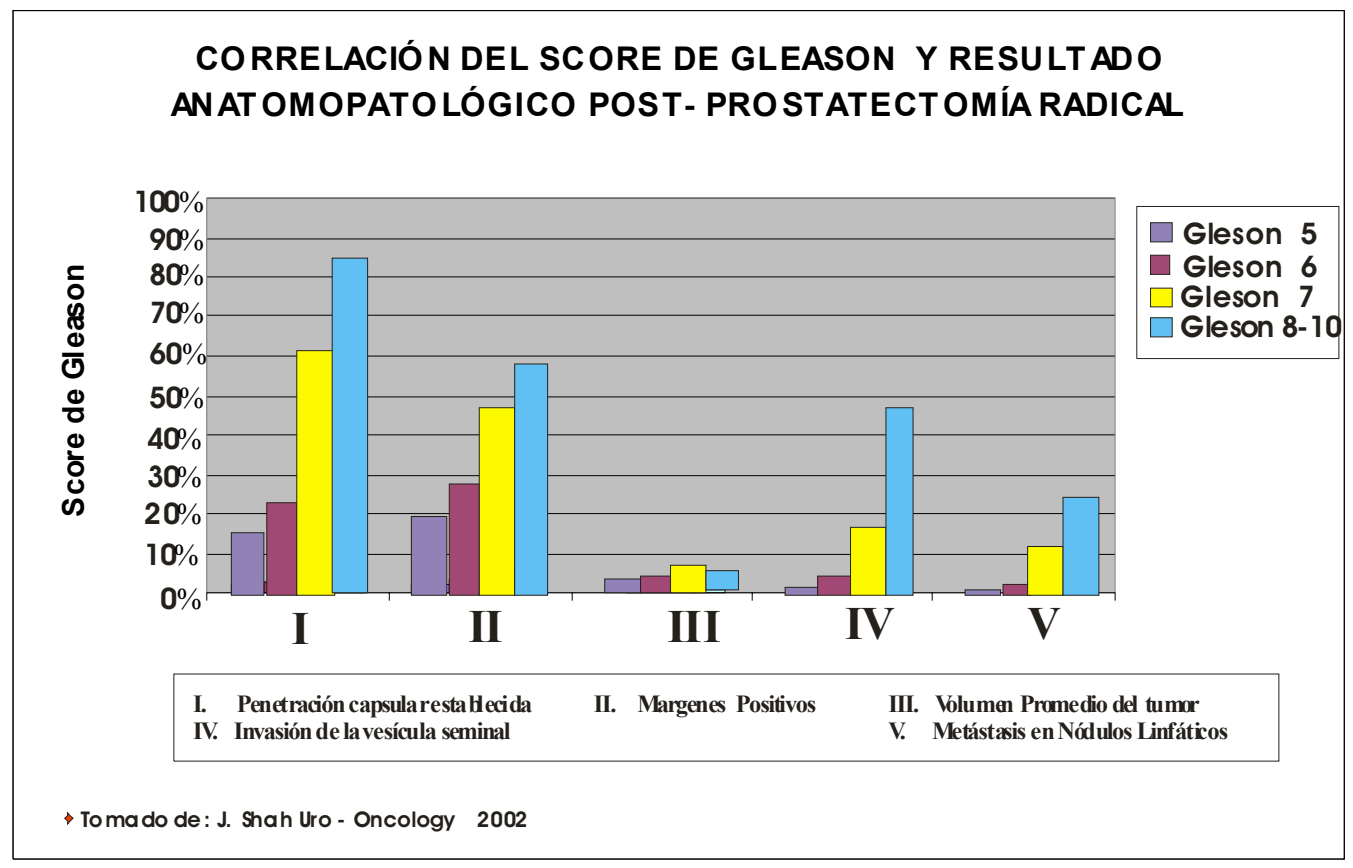

Figura $N^{\circ}$ 2. Score de Gleason y probabilidad de progresión local a 10 años.

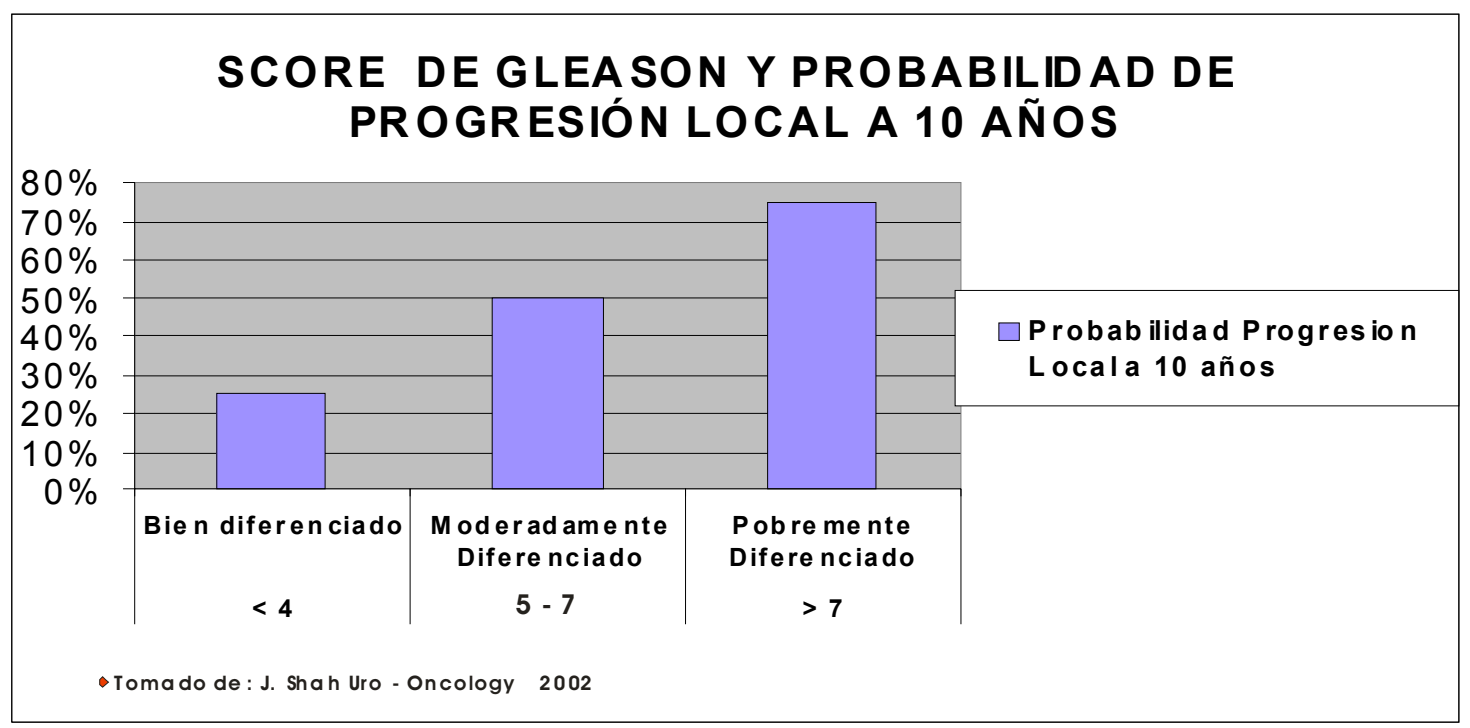

$<$ 7). En la práctica, se acepta para tumores de bajo riesgo una tasa APE inferior a 10, un número de biopsias positivas inferiores al 50\% y un resultado Gleason inferior a 7 .

Retomando las tablas de Partin, describimos 2 ejemplos de pacientes de Shah (4): 1) Un hombre de 55 años de edad, se ha sometido a biopsias de próstata ante la elevación en 1 año de su APE de 2ng/ml a 4ng/ $\mathrm{ml}, 2$ biopsias sobre 6 son positivas con un Gleason $=$ 9: en este hombre la prostatectomía radical tiene pocas posibilidades de ser curativa ya que el riesgo de penetración capsular es de $85 \%$ y el de márgenes positivos es de 60\%. 2) Un hombre de 65 años asintomático pero que presenta una tasa de APE de 7ng (T1c clínico), las biopsias de próstata descubren un cáncer con un Gleason =7: las posibilidades de que el paciente tenga un cáncer localizado de glándula luego de una prostatectomía radical, son sólo de $37 \%$ mientras que el riesgo de extensión extraprostática asciende de 40-54\% y el de infiltración de las vesículas seminales asciende a $12 \%$ (Figura $\mathrm{N}^{\circ} 1$ y figura $\mathrm{N}^{\circ} 2$ ). 
Ante estas constataciones, los urólogos tuvieron que aceptar que para hablar de cáncer localizado en la glándula se requería que el paciente presente simultáneamente un cáncer de estadío clínico T1 - T2 y un tumor de bajo riesgo. Para encontrar pacientes compatibles con esta definición, se imponía la necesidad de reducir el umbral de APE por debajo de 4ng/ml para realizar biopsias de próstata con riesgo a descubrir tumores no significativos o que no amenacen en absoluto al paciente.

\section{Del cáncer localizado a los tumores latentes (o sin consecuencias clínicas)}

En todas las series de autopsias, se ha demostrado que por encima de los 50 años de edad, del 30 a 50\% de hombres presentan focos de cáncer de próstata, pero el riesgo de que un hombre sufra algún día de cáncer de próstata clínicamente significativo, es inferior a $12 \%$ y de que muera es menos del $4 \%$.

E1 30\% de hombres en Europa son portadores de cáncer de próstata pero sólo $10 \%$ desarrollan la enfermedad durante su tiempo de vida. El número no va a disminuir, ya que la edad es el factor de riesgo más importante y la elevación de la expectativa de vida está en constante expansión (77 años para el hombre en Francia en 2004).

El riesgo de sobre detección del cáncer de próstata ha aumentado en estos últimos años por el hecho de que los urólogos por lo general, han bajado la tasa de APE para practicar biopsias (pasando a más de 3ng/ ml) y han aumentado el número de extracciones (pasando de 6 a 12, incluso 18) (5).

El examen de las piezas operatorias muestran en diversas instituciones que más del $25 \%$ de los tumores extraídos hoy en día no son significativos (criterios de Epstein). Para estos tumores infraclínicos, los resultados a 10 años, cualquiera que sea el tratamiento: observación activa, prostatectomía radical o radioterapia (externa o crioterapia), son equivalentes (Tabla $\mathrm{N}^{\circ} 2$ ).

Estudios recientes sugieren que una tasa de APE $=$ 2,5 $\mathrm{ng} / \mathrm{ml}$ tendría el mismo valor predictivo de cáncer que una tasa de APE $>4 \mathrm{ng} / \mathrm{ml}$. Thompson (6) utilizó una cohorte de individuos incluidos en el brazo placebo del estudio Prostate Cancer Prevention Trial (PCPT) reclutando a 18882 hombres de 55 años o más que presentaban una tasa de APE $<3 \mathrm{ng} / \mathrm{ml}$ y un tacto rectal normal. Nueve mil cuatrocientos cincuentainueve hombres fueron simplemente observados durante 7 años, los otros recibieron tratamiento con Finasteride. Dos mil novecientos cincuenta hombres conservaron una tasa de APE < 4ng durante la observación y aceptaron una biopsia de próstata después de 7 años. Entre 7 a $27 \%$ de ellos presentaron células neoplásicas en la próstata (Tabla $\mathrm{N}^{\circ} 3$ ). El autor se cuestiona entonces sobre el riesgo de sobre diagnóstico disminuyendo el umbral del APE con un riesgo de sobre tratamiento en lesiones no significativas.

El estudio N4 del Grupo de Cáncer de Próstata Escandinavo, analizó una cohorte de 654 pacientes observados durante 21 años y tratados solamente

Tabla $N^{\circ}$ 2. Cancer localizado de próstata, tratamiento y resultado a 10 años.

\begin{tabular}{llcc}
\hline $\begin{array}{c}\text { Cáncer de próstata } \\
\text { clínicamente } \\
\text { localizado }\end{array}$ & $\begin{array}{l}\text { Prostatectomía } \\
\text { radical }\end{array}$ & Rt. & $\begin{array}{c}\text { Vigilancia y } \\
\text { tratamiento diferido }\end{array}$ \\
\hline $\begin{array}{l}\text { Enfermedad-Específica } \\
10 \text {-años sobrevida }\end{array}$ & $88,5-93 \%$ & $66-74 \%$ & $80-85 \%$ \\
\hline Rt : Radioterapia & & & \\
\hline
\end{tabular}

Tabla $N^{\circ} 3$. Test de predicción para cancer de prostata.

\begin{tabular}{ccc}
\hline $\begin{array}{c}\text { Tasa de APE } \\
(\mathbf{n g} / \mathbf{m l})\end{array}$ & $\begin{array}{c}\mathbf{N}^{\circ} \text { Individuos } \\
(\mathbf{n}=\mathbf{2 , 9 5 0 )}\end{array}$ & $\begin{array}{c}\text { Pacientes con } \\
\text { Cáncer de Próstata } \\
(\mathbf{n}=\mathbf{4 4 9 )}\end{array}$ \\
\hline$\leq 0,5$ & 486 & $32(6,6 \%)$ \\
$0,6-1,0$ & 791 & $80(10,1 \%)$ \\
$1,1-2,0$ & 998 & $170(17,0 \%)$ \\
$2,1-3,0$ & 482 & $115(23,9 \%)$ \\
$3,1-4,0$ & 193 & $52(26,9 \%)$ \\
\hline
\end{tabular}


cuando era necesario (tratamiento diferido). En esta cohorte, a un subgrupo de 223 pacientes se le descubrió cáncer después de una resección endoscópica o una anomalía al tacto rectal (estadío clínico T1 o T2), 203 fallecieron a lo largo de la observación $(91 \%)$ pero sólo 35 (16\% de la cohorte) murieron de cáncer de próstata. $\mathrm{Si}$ admitimos que la prostatectomía radical permite prevenir $50 \%$ de decesos por cáncer de próstata, se puede demostrar que en este grupo de enfermos, la prostatectomía hubiera beneficiado a 18 pacientes $(8 \%)$ y no habría tenido ninguna utilidad en los otros 205 (2).

Un estudio de fase II procedente de Canadá sobre una cohorte de 299 pacientes portadores de un cáncer de próstata de bajo riesgo, propuso una observación activa con intervención diferida sólo en el caso que el tiempo de duplicación de APE apareciera después de 2 años de observación. Todos los pacientes tenían más de 70 años. La mayoría permaneció bajo observación (sometiendo a 24 pacientes a prostatectomía radical diferida). A los 8 años, la supervivencia actuarial era de $85 \%$ y la supervivencia relacionada al cáncer era de $99 \%$, el autor concluye que la mayor parte de hombres con cáncer de próstata de riesgo favorable morirá de otra patología ajena al cáncer (8).

\section{DISCUSIÓN Y CONCLUSIÓN}

La historia natural del cáncer de próstata se conoce mejor gracias a los estudios que han podido realizarse tanto en epidemiología como por medio de cohortes de pacientes observados u operados por prostatectomía radical.

En el siglo XX, los esfuerzos en salud pública se inclinaron hacia la prolongación de la duración de la vida, hecho que se convirtió en realidad y la promesa de curar este cáncer, lo que no se pudo concretar. El siglo XXI tiene otros objetivos: la calidad de vida que ahora debe acompañar a la prolongación de la vida y para el cáncer, un mejor control del mismo antes que su misma curación.

Todos los últimos trabajos confirman que sólo el cáncer localizado en la glándula prostática es quirúrgicamente curable $y$, sólo los de bajo riesgo tienen una posibilidad real de limitarse a la glándula. En este grupo de pacientes con cáncer localizado, muchos no son de importancia clínica. Gran parte de los pacientes portadores de células neoplásicas en la próstata morirán de otra causa sin necesidad de que hayan sufrido de la próstata.

Este trabajo resalta los límites de la cirugía y la actual falta de criterios que permitan conocer el carácter localizado de la lesión en la glándula y su agresividad, asi como la falta de tratamientos alternativos eficaces en caso de lesión extraprostática.

Finalmente, es meritorio querer diagnosticar precozmente el cáncer de próstata, sin embargo, desde el momento en que éste es anunciado al paciente, la palabra " cáncer » es devastadora y su anuncio transforma la vida del paciente, trayéndole sensación de muerte y pudiéndolo llevar a un desamparo psicológico.

A los urólogos decirles que no sobreestimen el cáncer y no lo sobre traten con intervenciones que alteren la calidad de vida: la prostatectomía radical provoca incontinencia urinaria entre $5-20 \%$ y en 40 a $70 \%$, disfunción eréctil. También expresarles que no hagan que el paciente pierda la oportunidad por un diagnóstico tardío.

¿Existe el cáncer de próstata localizado a la glándula? la pregunta se presenta ahora entre el cáncer diagnosticado mientras no es significativo y el que ya sobrepasó la cápsula.

\section{Correspondencia:}

Luis Zegarra Montes

Departamento de Cirugía

Universidad Peruana Cayetano heredia

Av Honorio Delgado 430

San Martin de Porres

Lima Perú

\section{REFERENCIAS BIBLIOGRÁFICAS}

1. Epstein JL, Walsh PC, Carmichael M, Brendler CB. Pathologic and clinical findings to predict tumor extent of non-palpable (stage T1c) prostate cancer. JAMA 1994; 271:368-74.

2. Holmberg L, Bill-Axelson A, et al. A randomized trial comparing radical prostatectomy with watchful waiting in early prostate cancer. N Engl J Med 2002; 347: 781-89.

3. Partin AW, Kattan MW, Subong EP, Walsh PC, Oesterling JE, Scardino PT, et al. Combination of prostate-specific antigen, clinical stage, and Gleason score to predict pathological stage of localized prostate cancer: a multi-institutional update. JAMA 1997; 277: 1445-49.

4. Shah J. Day 2 Uro-oncology. Barnham: Eurocommunica Publications, 2002.

5. Murphy AM, McKiernan JM, Olsson CA. Controversies in prostate cancer screening. J Urol 2004; $172: 1822-24$

6. Thompson MI, Pauler DK, Goodman PJ, Tangen CM, Lucia MC, Parnes HL, et al. Prevalence of prostate 
cancer among men with a prostate-specific antigen level £ 4.0 ng per milliliter. N Engl J Med 2004; 350 : 2239-46.

7. Polascik TJ, Pearson JD, Partin AW, Polascik DT. Use of multivariate models to improve prediction of pathologic stage for men with clinically localized prostate cancer. Prostate Cancer Prostatic Dis 1998; 1(6): 301-06.

8. Klotz L. Active surveillance with selective delayed intervention: using natural history to guide treatment in good risk prostate cancer. J Urol 2004; 172 : 48-51. 